\title{
MODULAR SUPERVISORY CONTROL OF A CLASS OF CONCURRENT DISCRETE EVENT SYSTEMS
}

\author{
B. Gaudin * H. Marchand *
}

* Irisa, Campus universitaire de Beaulieu, Rennes.

\begin{abstract}
In this paper, we are interested in the control of a particular class of Concurrent Discrete Event Systems defined by a collection of components that interact with each other. We investigate the computation of the supremal controllable language contained in the one of the specification. We do not adopt the decentralized approach. Instead, we have chosen to perform the control on some approximations of the plant derived from the behavior of each component. The behavior of these approximations is restricted so that they respect a new language property for discrete event systems called partial controllability condition that depends on the specification. It is shown that, under some assumptions, the intersection of these "controlled approximations" corresponds to the supremal controllable language contained in the specification with respect to the plant. This computation is performed without having to build the whole plant, hence avoiding the state space explosion induced by the concurrent nature of the plant.
\end{abstract}

Keywords: Discrete Event Systems, Supervision and control, Concurrent Systems, Partial controllability.

\section{INTRODUCTION}

In this paper, we are interested in the control of Concurrent Discrete Event Systems defined by a collection of components that interact with each other. Supervisory control [Ramadge and Wonham (1989)] consists in modifying a system (plant) such that the modified (or controlled) system satisfies a given property (or specification). Given a plant and a specification modeled by languages, in the Ramadge \& Wonham theory, one important phase is the computation of the supremal controllable sub-language contained in a language that represents the expected behavior. However, although this computation is polynomial in the number of states of the plant and of the specification, it is well known that the plant size grows exponentially with the number of components that compose the plant. This renders the computation of the supervisors not practical because of the size of the generated state space which is often too important when dealing with large scale systems.

Several approaches have been recently investigated to deal with the complexity issue of the control of Concurrent Discrete Event Systems. Given a Concurrent Discrete Event System $G=G_{1}\|\cdots\| G_{n}$ and a specification expressed by a language $K$, the problem is to compute the supremal controllable sublanguage of $K \cap \mathcal{L}(G)$ w.r.t. $\mathcal{L}(G)$ without having to build $\mathcal{L}(G)$. In [deQueiroz and Cury (2000); Akesson et al. (2002)], the authors consider the control of a product plant (i.e. systems composed of asynchronous subsystems, not sharing common events) ${ }^{1}$. Given a specification, a local system is built from the components that are coordinated by the specification (i.e. all the components that share some events used to express it). It is then sufficient to compute the local supervisor ensuring the specification with respect to this local system in order to obtain the result on the whole system. Closely related to the decentralized theory, under the hypothesis that the specification is separable and that the shared events are controllable, the authors of [Willner and Heymann (1991)] provide a solution allowing to compute local modular supervisors $\mathcal{S}_{i}$ acting upon $G_{i}$ and to operate the individually controlled plant $\mathcal{S}_{i} / G_{i}$ concurrently in such a way that the behavior of the controlled plant (i.e. $\mathcal{L}\left(\|_{i} \mathcal{S}_{i} / G_{i}\right)$ ) corresponds to the supremal controllable sublanguage of $K$ w.r.t. the plant $\mathcal{L}(G)$. The same methodology has been used in [Rohloff and Lafortune (2003)] for the control of concurrent plant for which the various components have an identical structure and under the constraints that the local supervisors $\mathcal{S}_{i}$ are only operating on a subset of the local events. See also [Abdelwahed and Wonham (2002); Jiang and Kumar (2000); Leduc et al. (2001)] for other works relating to the control of concurrent plant.

In this paper, compared to [Willner and Heymann (1991)], we adopt a dual approach. Instead of having one local supervisor per component that enforces local control actions with respect to the events of this component, we have chosen to perform the control on some approximations of the plant derived from the behavior of

\footnotetext{
1 they actually first transform a concurrent plant in a modular plant.
} 
each component. The behavior of these approximations is restricted so that they respect a new language property for discrete event systems called partial controllability condition that depends on $K$. Under some assumptions, it is shown that a supervisor can be derived from these "controlled approximations" such that the behavior of the controlled plant corresponds to the supremal controllable language contained in $K$ w.r.t. the plant $G$. More details (and missing proofs) are available in [Gaudin and Marchand (2004)].

\section{PRELIMINARIES}

Model and Supervisory Control overview. The basic structures from which the plant is built are Finite State Machines (FSM), that are defined by a 4-tuple $G=$ $\left\langle\Sigma, \mathcal{X}, x_{o}, \delta\right\rangle$, where $\Sigma$ is the finite alphabet of $G$. $\mathcal{X}$ is the finite set of states, $x_{o} \in \mathcal{X}$ is the initial state, whereas $\delta$ is the partial transition function defined over $\Sigma \times \mathcal{X} \longrightarrow \mathcal{X}$. The notation $\delta(\sigma, x)$ ! means that $\delta(\sigma, x)$ is defined, i.e., there is a transition labeled by an event $\sigma$ out of state $x$ in machine $G$. Likewise, for $x \in \mathcal{X}$ and $s \in \Sigma^{*}, \delta(s, x)$ denotes the state reached by taking the sequence of events defined by trace $s$ from state $x$ in machine $G$. The behavior of the system is described by the language $\mathcal{L}(G) \subseteq \Sigma^{*}$ generated by $G$. (i.e. $\mathcal{L}(G)=\left\{s \in \Sigma^{*} \mid \delta\left(s, x_{o}\right) !\right\}$.

Given $s, s^{\prime} \in \Sigma^{*}$, we say that $s^{\prime} \leq s$ whenever $s^{\prime}$ is a prefix of $s$ (i.e. it exists $t \in \Sigma^{*}$ s.t. $s=s^{\prime} t$ ). We denote by $\bar{L}$ the prefix-closure of a language $L \subseteq \Sigma^{*}$ $\left(\bar{L}=\left\{s \in \Sigma^{*} \mid \exists s^{\prime} \in L, s \leq s^{\prime}\right\}\right)$. Note that $\overline{\mathcal{L}}(G)$, as defined above, is prefix-closed (i.e. $\mathcal{L}(G)=\overline{\mathcal{L}(G)}$ ). For $L \subseteq \Sigma^{*}$ and $\Sigma^{\prime} \subseteq \Sigma$, we use $L\left(s, \Sigma^{\prime}\right)$ to denote the set of suffixes of $L$ after $s$ that belongs to $\Sigma^{\prime *}$, i.e. $L\left(s, \Sigma^{\prime}\right)=\left\{t \in \Sigma^{\prime *} \mid s t \in L\right\}$.

Given a plant to be controlled, some of its events in $\Sigma$ are said to be uncontrollable $\left(\Sigma_{u c}\right)$, i.e., their occurrence cannot be prevented by the supervisor, while the others are controllable $\left(\Sigma_{c}\right)$. First, we recall the definition of a controllable language [Ramadge and Wonham (1989)].

Definition 1. Let $G$ be an FSM modeling the plant and $K \subseteq \mathcal{L}(G)$ the prefix-closed specification. Then $K$ is controllable with respect to $\Sigma_{u c}$ and $G$ (or $\mathcal{L}(G)$ ) if $K \Sigma_{u c} \cap \mathcal{L}(G) \subseteq K$

We denote by $K^{\uparrow L, c}$ or $\operatorname{Sup} C\left(K, L, \Sigma_{u c}\right)$ the supremal controllable sub-language of $K$ w.r.t. $\Sigma_{u c}$ and $\mathcal{L}(G)=$ $L$ (see [Ramadge and Wonham (1989)]).

In some situations, it is also of interest to compute $K^{\downarrow L, c}$ the infimal prefix-closed and controllable superlanguage of $K$ w.r.t. $\mathcal{L}(G)$ and $\Sigma_{u c}$, which basically corresponds to the smallest prefix-closed language that contains $K$ and that is controllable w.r.t. $\Sigma_{u c}$ and $\mathcal{L}(G)=L$. It can be shown (see e.g. [Cassandras and Lafortune (1999)]) that $K^{\downarrow L, c}=K \Sigma_{u c}^{*} \cap L$.

Concurrent DES. In this paper, a plant is composed of several components, sharing common events, i.e. a plant $G$ is modeled as a collection of FSM $G_{i}=$ $\left\langle\Sigma_{i}, \mathcal{X}_{i}, x_{o i}, \delta_{i}\right\rangle$. The global behavior of the plant is given by $G=G_{1}\|\cdots\| G_{n}$, where the operation
$\|$ is the classical parallel composition (i.e. $G_{1} \| G_{2}$ represents the concurrent behavior of $G_{1}$ and $G_{2}$ with synchronization on the shared events). Now, given the set of FSM $\left(G_{i}\right)_{i \leq n}$ modeling $G$, we denote by $\Sigma_{s}$ the set of shared events of $G$, i.e

$$
\Sigma_{s}=\left\{\sigma \in \Sigma \mid \exists i \neq j, \sigma \in \Sigma_{i} \cap \Sigma_{j}\right\} .
$$

Let $\Sigma^{\prime} \subseteq \Sigma$, then $P_{\Sigma^{\prime}}: \Sigma^{*} \rightarrow \Sigma^{* *}$ is the natural projection from $\Sigma^{*}$ to $\Sigma^{\prime *}$ that erases in a sequence of $\Sigma^{*}$ all the events that do not belong to $\Sigma^{\prime}$.This definition is easily extended to the projection of regular languages as follows: $P_{\Sigma^{\prime}}(L)=\left\{s^{\prime} \in \Sigma^{\prime *} \mid \exists s \in L, s^{\prime}=P_{\Sigma^{\prime}}(s)\right\}$. Given $L \subseteq \Sigma^{\prime *} \subseteq \Sigma^{*}$, the inverse projection is defined by $P_{\Sigma^{\prime}}^{-1}(\bar{L})=\left\{s \in \Sigma^{*} \mid P_{\Sigma^{\prime}}(s) \in L\right\}$. From an implementation point of view, if $H$ denotes the FSM such that $\mathcal{L}(H)=L$, then the FSM modeling the inverse projection of $L$, noted $H^{-1}$, can be obtained from $H$ by simply adding self-loops labeled by events in $\Sigma \backslash \Sigma^{\prime}$ to each state of $H$.

Given a discrete event system $G=G_{1}\|\cdots\| G_{n}$, with $\mathcal{L}\left(G_{i}\right) \subseteq \Sigma_{i}^{*}$, we simply denote by $P_{i}$ the projection from $\Sigma^{*}$ to $\Sigma_{i}^{*}$ and by $P_{i}^{-1}$ the inverse projection from $\Sigma_{i}^{*}$ to $\Sigma^{*}$. Based on these operations, the language resulting from the parallel composition of FSM is characterized by:

$$
\mathcal{L}(G)=P_{1}^{-1}\left[\mathcal{L}\left(G_{1}\right)\right] \cap \cdots \cap P_{n}^{-1}\left[\mathcal{L}\left(G_{n}\right)\right]
$$

The following technical lemmas will be useful.

Lemma 1. [Gaudin and Marchand (2004)] Let $L \subseteq \Sigma^{\prime *}$ and $\Sigma^{\prime} \subseteq \Sigma$, let $s \in L$ and $s^{\prime} \in \Sigma^{*}$, then $s s^{\prime} \in$ $P_{\Sigma^{\prime}}^{-1}(L) \Longrightarrow s P_{\Sigma^{\prime}}\left(s^{\prime}\right) \in P_{\Sigma^{\prime}}^{-1}(L)$.

Lemma 2. ((3.1) of [Willner and Heymann (1991)]) Let $G=G_{1}\|\cdots\| G_{n}, s \in \mathcal{L}(G), i \in\{1, \ldots, n\}$ and $\sigma \in \Sigma_{i} \backslash \Sigma_{s}$. Then $s \sigma \in \mathcal{L}(G) \Longleftrightarrow s \sigma \in P_{i}^{-1}\left(\mathcal{L}\left(G_{i}\right)\right)$.

$\diamond$

Control Problem formulation \& Related Works Let $G=G_{1}\|\cdots\| G_{n}$ be the plant to be controlled and $L_{i}=\mathcal{L}\left(G_{i}\right)$ be the language generated by the component $G_{i}$ for $i \leq n$. The alphabet of $G_{i}$ is partitioned into the controllable event set $\Sigma_{i, c}$ and the uncontrollable event set $\Sigma_{i, u c}$, i.e. $\Sigma_{i}=\Sigma_{i, u c} \cup \Sigma_{i, c}$. The alphabet of the global plant $G$ is given by:

$$
\Sigma=\bigcup_{i} \Sigma_{i}, \Sigma_{c}=\bigcup_{i} \Sigma_{i, c}, \text { and } \Sigma_{u c}=\Sigma \backslash \Sigma_{c} .
$$

Moreover, we assume that the following relation holds between the control status of shared events:

$$
\forall i, j, \Sigma_{i, u c} \cap \Sigma_{j, c}=\emptyset
$$

which simply means that the components that share an event agree on the control status of this event. Under this hypothesis, we have that

$$
\Sigma_{u c}=\cup_{i} \Sigma_{i, u c}
$$

Let $K \subseteq \Sigma^{*}$ be the expected behavior. The problem, we are interested in, is the Basic Supervisory Control Problem, i.e . the problem is to compute the supremal controllable sublanguage $(K \cap \mathcal{L}(G))^{\uparrow c}$ of $K \cap \mathcal{L}(G)$ 
w.r.t. $\mathcal{L}(G)$. However, knowing that the synthesis algorithms are polynomial in the number of states of $G$ and that the size of the state space of $G$ is exponential in the number of components of $G$, it is important to design algorithms that compute the controller by taking advantage of the structure of $G$ without building it. Hence, the actual problem is to compute $(K \cap \mathcal{L}(G))^{\uparrow c}$ without computing neither $\mathcal{L}(G)$ nor $K \cap \mathcal{L}(G)$.

A Decentralized approach: The works of [Willner and Heymann (1991)] is closely related to the decentralized theory. The authors consider the control of Concurrent DES $G_{1}\|\cdots\| G_{n}$. Given a language-based specification $K$, they provide some solutions allowing to compute local modular supervisors $\mathcal{S}_{i}$ on $G_{i}$ (based on a notion of separable specification (See Definition 2)) and to operate the individually controlled system $\mathcal{S}_{i} / G_{i}$ concurrently in such a way that the controlled behavior corresponds to the supremal controllable sublanguage of $K \cap \mathcal{L}(G)$ w.r.t. $\mathcal{L}(G)$.

Definition 2. $\mathcal{L} \subseteq \Sigma^{*}$ is said to be separable w.r.t. $\left\{\Sigma_{i}\right\}_{i \leq n}$ with $\cup_{i \leq n} \Sigma_{i}=\Sigma$, whenever there exists a set of languages $\left\{\mathcal{L}_{i}\right\}_{i \leq n}$ (called generating set), s.t. $\mathcal{L}_{i} \subseteq \Sigma_{i}^{*}$ and $\mathcal{L}=\mathcal{L}_{1}\|\cdots\| \mathcal{L}_{n}=\cap_{i} P_{i}^{-1}\left(\mathcal{L}_{1}\right)$

Based on this definition, [Willner and Heymann (1991)] shown that

Theorem 1. Let $G=G_{1}\|\cdots\| G_{n}$, with $\mathcal{L}\left(G_{i}\right) \subseteq \Sigma_{i}^{*}$. and $K$ the expected specification. If $\Sigma_{s} \subseteq \Sigma_{c}$ and $K$ is separable w.r.t. $\left\{\Sigma_{i}\right\}_{i \leq n}$, then

$$
\begin{aligned}
& \|_{i \leq n} \operatorname{Sup} C\left(P_{i}(K)\right.\left.\cap \mathcal{L}\left(G_{i}\right), \mathcal{L}\left(G_{i}\right), \Sigma_{i, u c}\right)= \\
& \operatorname{Sup} C\left(K \cap \mathcal{L}(G), \mathcal{L}(G), \Sigma_{u c}\right) \diamond
\end{aligned}
$$

Hence, given a Concurrent DES $G$ and a separable specification $K$, Theorem 1 shows that there exists a set of supervisors $\mathcal{S}_{i}$ acting upon $G_{i}$, such that $\|_{i \leq n}$ $\mathcal{L}\left(S_{i} / G_{i}\right)=(K \cap \mathcal{L}(G))^{\uparrow c}$.

If $K$ is separable w.r.t. $\left\{\Sigma_{i}\right\}_{i \leq n}$ (which can be checked in $\mathcal{O}\left(m^{n+1}\right)$, where $m$ is the size of the FSM that generates $K$ ), then synthesizing the local supervisors requires the computation of the projection of $K$ over $\Sigma_{i}$. In the worst case, the size of the FSM that generates $P_{i}(K)$ is in $\mathcal{O}\left(2^{m}\right)$. Hence, solving the supervisory control problem will require $\mathcal{O}\left(n .2^{m} . N\right)$ space where $N$ is the size of each component.

Our approach: Our approach is different and is more related to the modular approach of [Wonham and Ramadge (1988)]. Indeed, the plant $G$ can be described by the following parallel composition of FSM $G=\|_{i \leq n}$ $G_{i}^{-1}$, where $G_{i}^{-1}$ is the FSM such that $\mathcal{L}\left(G_{i}^{-1}\right)=$ $P_{i}^{-1}\left(\mathcal{L}\left(G_{i}\right)\right)$. In fact, each $G_{i}^{-1}$ can be seen as an approximation of the plant $G$ to be controlled.

Compared to [Willner and Heymann (1991)], we adopt a dual approach. Instead of controlling each component $G_{i}$ (i.e. $\mathcal{L}\left(G_{i}\right)$ ) to enforce $P_{i}(K)$, we have chosen to control the approximations $\mathcal{L}\left(G_{i}^{-1}\right)$ of the plant in order to enforce $K$. However, it is not sufficient to compute a supervisor $\mathcal{S}_{i}$ acting upon $G_{i}^{-1}$ that restricts the behavior $\mathcal{L}\left(G_{i}^{-1}\right)$ to the supremal controllable sublanguage of $K \cap \mathcal{L}\left(G_{i}^{-1}\right)$ and to operate the controlled systems $\mathcal{S}_{i} / G_{i}^{-1}$ concurrently to obtain the supremal controllable sublanguage of $K \cap \mathcal{L}(G)$ (the result may be not supremal). So the idea of our method is to refine the notion of controllability.

The property that we ensure on each $G_{i}^{-1}$ according to $K$ is called the partial controllability condition and is defined in Section 3. As in the case of the controllability concept, it will be shown that there exists a supremal partially controllable sublanguage of $K \cap \mathcal{L}\left(G_{i}^{-1}\right)$ w.r.t. $K$ and $G_{i}^{-1}$, called $K_{i}^{\uparrow p c}$. It is then shown that $\cap_{i \leq n} K_{i}^{\uparrow p c} \subseteq(K \cap \mathcal{L}(G))^{\uparrow c}$ (Theorem 2) and that under some conditions on $K$ the equality holds (Theorem 3 and 4). A comparison with the results of [Willner and Heymann (1991)] is then done.

\section{PARTIAL CONTROLLABILITY PROPERTY}

In this section, we introduce a new concept of controllability, named Partial Controllability, that will serve as the bases of the modular computation of supervisors for Concurrent discrete event systems.

\subsection{Definition and useful properties}

Definition 3. Let $M \subseteq L \subseteq \Sigma^{*}$ be prefix-closed languages. Let $\Sigma_{u c}^{\prime} \subseteq \Sigma_{u c} \subseteq \Sigma$ be two sub-alphabets of $\Sigma$. Let $M^{\prime} \subseteq M$ be a prefix-closed language. $M^{\prime}$ is partially controllable with respect to $\Sigma_{u c}^{\prime}, \Sigma_{u c}, M$ and $L$ if

(i) $M^{\prime}$ is controllable w.r.t $\Sigma_{u c}^{\prime}$ and $L$.

(ii) $M^{\prime}$ is controllable w.r.t $\Sigma_{u c}$ and $M$.

In general, $M$ is not partially controllable with respect to $\Sigma_{u c}^{\prime}, \Sigma_{u c}, M$ and $L$ (e.g. if $M$ is not controllable w.r.t. $\Sigma_{u c}^{\prime}$ and $\left.L\right)$. However, it can be shown that there exists a supremal sub-language of $M$ that has this property.

\section{Proposition 1. [Gaudin and Marchand (2004)]}

Let $M \subseteq L \subseteq \Sigma^{*}$ be prefix-closed languages, $\Sigma_{u c}^{\prime} \subseteq$ $\Sigma_{u c}$. There exists a unique supremal language, denoted by $M^{\uparrow p c}$, which is partially controllable w.r.t $\Sigma_{u c}^{\prime}$, $\Sigma_{u c}, M$ and $L$. Moreover

$M^{\uparrow p c}=\overline{M^{\uparrow p c}}=\operatorname{SupC}\left(\operatorname{SupC}\left(M, \Sigma_{u c}^{\prime}, L\right), \Sigma_{u c}, M\right) \diamond$

Prop. 1 offers a practical way to compute the supremal partially controllable sub-language of $M$ w.r.t. to $\Sigma_{u c}^{\prime}$, $\Sigma_{u c}$, and $L$. It can be shown that its computation is in $\mathcal{O}\left(|\Sigma| N_{M}^{2} N_{L}\right)$, where $N_{M}$ is the size of the FSM encoding $M$ and $N_{L}$ the one of $L$.

\section{CONTROL OF CONCURRENT DES}

Given a Concurrent DES, $G=G_{1}\|\cdots\| G_{n}$, and a control objective $K$, we want to compute a controllable sub-language of $K \cap \mathcal{L}(G)$ w.r.t. $\mathcal{L}(G)$ and $\Sigma_{u c}$, without having to build $G$ itself.

\subsection{Modular computation of a controllable sub-language of $K$ w.r.t. $\mathcal{L}(G)$}

Based on the concept of partial controllability applied on $K$ and on the approximations of the plant $P_{i}^{-1}\left(\mathcal{L}\left(G_{i}\right)\right)$ 
derived from each of its components, the next theorem provides a modular way to compute a sub-language of $K$ that is controllable with respect to the plant.

Theorem 2. Let $G=G_{1}\|\cdots\| G_{n}$, with $G_{i}$ acting upon $\Sigma_{i}=\Sigma_{i, u c} \cup \Sigma_{i, c}$ and $L_{i}=\mathcal{L}\left(G_{i}\right)$. Let $K \subseteq$ $\Sigma^{*}$ be a prefix-closed language modeling the expected behavior. For $i \leq n$, we note

- $K_{i}=K \cap P_{i}^{-1}\left(\mathcal{L}\left(G_{i}\right)\right)$, and

- $K_{i}^{\uparrow p c}$ the supremal sublanguage of $K_{i}$ partially controllable w.r.t. $\Sigma_{i, u c}, \Sigma_{u c}, K_{i}$ and $P_{i}^{-1}\left(\mathcal{L}\left(G_{i}\right)\right)$.

Then, $\bigcap_{i \leq n} K_{i}^{\uparrow p c}$ is controllable w.r.t. $\Sigma_{u c}$ and $\mathcal{L}(G)$.

Proof : First, as $K, \mathcal{L}\left(G_{i}\right)$ are prefix-closed, languages $P_{i}^{-1}\left(\mathcal{L}\left(G_{i}\right)\right)$ for $i \leq n$ are prefix-closed, and therefore $K_{i}$ and $K_{i}^{\uparrow p c}$ are also prefix-closed. Now, according to Definition 1, we have to show that

$$
\left(\bigcap_{i \leq n} K_{i}^{\uparrow p c}\right) \Sigma_{u c} \cap \mathcal{L}(G) \subseteq \bigcap_{i \leq n} K_{i}^{\uparrow p c}
$$

Let $s \in \bigcap_{i \leq n} K_{i}^{\uparrow p c}$ and $\sigma \in \Sigma_{u c}$ be such that $s . \sigma \in$ $\mathcal{L}(G)$. We thus have to show that $s \sigma \in \bigcap_{i<n} K_{i}^{\uparrow p c}$. Without lost of generality we can assume that $\sigma \in \Sigma_{1, u c}$.

Since $s \sigma \in \mathcal{L}(G), s \sigma \in P_{1}^{-1}\left(\mathcal{L}\left(G_{1}\right)\right)$. Hence we have that $s \sigma \in K_{1}^{\uparrow p c} \Sigma_{1, u c} \cap P_{1}^{-1}\left(\mathcal{L}\left(G_{1}\right)\right)$. Moreover, according to definition $3, K_{1}^{\uparrow p c}$ is controllable w.r.t. $\Sigma_{1, u c}$ and $P_{1}^{-1}\left(\mathcal{L}\left(G_{1}\right)\right)$, from which we can conclude that $s \sigma \in K_{1}^{\uparrow p c}$.

As $s \sigma \in K_{1}^{\uparrow p c}$, we have that $s \sigma \in K$. And as $s \sigma \in$ $\mathcal{L}(G)$, then $\forall i \leq n, s \sigma \in P_{i}^{-1}\left(\mathcal{L}\left(G_{i}\right)\right)$, which entails that $\forall i \leq n, s \sigma \in K_{i}=K \cap P_{i}^{-1}\left(\mathcal{L}\left(G_{i}\right)\right)$. Hence, $\forall i \leq n, s \sigma \in K_{i}{ }^{\uparrow p c} \Sigma_{u c} \cap K_{i}$. Now, according to definition $3, \forall i \leq n, K_{i}^{\uparrow p c}$ is controllable w.r.t. $\Sigma_{u c}$ and $K_{i}$. Hence $\forall i \leq n, s \sigma \in K_{i}^{\uparrow p c}$, which entails that $s \sigma \in \bigcap_{i \leq n} K_{i}^{\uparrow p c}$.

\subsection{Computation of $\operatorname{Sup} C\left(\left(K \cap \mathcal{L}(G), \mathcal{L}(G), \Sigma_{u c}\right)\right.$}

In the remainder of this paper we use $(K \cap \mathcal{L}(G))^{\uparrow c}$ to denote $\operatorname{Sup} C\left(\left(K \cap \mathcal{L}(G), \mathcal{L}(G), \Sigma_{u c}\right)\right.$. According to Theorem 2, we have that $\bigcap_{i<n} K_{i}^{\uparrow p c} \subseteq(K \cap \mathcal{L}(G))^{\uparrow c}$. But, the equality does not hold in general. In this section, we present some conditions under which Theorem 2 gives access to the supremal solution.

Let us first introduce lemma 1. This lemma shows that whenever the shared events $\Sigma_{s}$ are controllable, then $(K \cap \mathcal{L}(G))^{\uparrow c}$ verifies a part of the partial controllability condition.

Lemma 1. Let $G=G_{1}\|\cdots\| G_{n}$ be the plant and $K \subseteq \Sigma^{*}$ a prefix-closed language modeling the expected behavior, then if $\Sigma_{s} \subseteq \Sigma_{c}$, then $\forall i \leq n$, $(K \cap \mathcal{L}(G))^{\uparrow c}$ is controllable w.r.t $\Sigma_{i, u c}, P_{i}^{-1}\left(\mathcal{L}\left(G_{i}\right)\right)$.

Proof : Let $i \leq n$. Let us consider $s \in(K \cap \mathcal{L}(G))^{\uparrow c}$ and $\sigma \in \Sigma_{i, u c}$ such that $s \sigma \in P_{i}^{-1}\left(\mathcal{L}\left(G_{i}\right)\right)$. We have to show that $s \sigma \in(K \cap \mathcal{L}(G))^{\uparrow c}$. Since $\sigma \in \Sigma_{i, u c}$ and $\Sigma_{s} \subseteq \Sigma_{c}$, we have $\sigma \in \Sigma_{i} \backslash \Sigma_{s}$. Moreover, $s \in P_{i}^{-1}\left(\mathcal{L}\left(G_{i}\right)\right), s \in \mathcal{L}(G)$ and $s \sigma \in P_{i}^{-1}\left(\mathcal{L}\left(G_{i}\right)\right)$, which entails that $s \sigma \in \mathcal{L}(G)$ (Lemma 2). Now, as $\Sigma_{i, u c} \subseteq \Sigma_{u c}$, we have that $s \sigma \in(K \cap \mathcal{L}(G))^{\uparrow c} . \Sigma_{u c} \cap$ $\mathcal{L}(G)$. Since $(K \cap \mathcal{L}(G))^{\uparrow c}$ is controllable w.r.t $\Sigma_{u c}$ and $\mathcal{L}(G)$, this entails that $s \sigma \in(K \cap \mathcal{L}(G))^{\uparrow c}$.

The next theorem states that whenever the shared event are controllable and the language of the control objective is included in the one of the plant then our methodology gives access to the supremal controllable sub-language of $K$ w.r.t. $L$ and $\Sigma_{u c}$.

Theorem 3. If $\Sigma_{s} \subseteq \Sigma_{c}$ and $K \subseteq \mathcal{L}(G)$, then with the notations of Theorem 2, $\bigcap_{i \leq n} K_{i}^{\uparrow p c}=K^{\uparrow c}$.

Proof : From Theorem 2, $\bigcap_{i \leq n} K_{i}^{\uparrow p c} \subseteq(K \cap \mathcal{L}(G))^{\uparrow c}$. As $K \cap \mathcal{L}(G)=K$ it is then sufficient to show that $K^{\uparrow c} \subseteq \bigcap_{i \leq n} K_{i}^{\uparrow p c}$ or, equivalently that $\forall i \leq n, K^{\uparrow c} \subseteq$ $K_{i}^{\uparrow p c}$. To do so, let us pick up a $i \leq n$, and let us show that $K^{\uparrow c}$ is partially controllable w.r.t. $\Sigma_{i, u c}, \Sigma_{u c}, K_{i}$ and $P_{i}^{-1}\left(L_{i}\right)$.

(i) First, according to lemma $1, K^{\uparrow c}$ is controllable w.r.t $\Sigma_{i, u c}$ and $P_{i}^{-1}\left(\mathcal{L}\left(G_{i}\right)\right)$.

(ii) Let us now show that $K^{\uparrow c}$ is controllable w.r.t. $\Sigma_{u c}$ and $K_{i}$. Let us consider $s \in K^{\uparrow c}, \sigma \in \Sigma_{u c}$ such that $s \sigma \in K_{i}$, we have to prove that $s \sigma \in K^{\uparrow c}$. Since $K_{i} \subseteq \mathcal{L}(G), s \sigma \in \mathcal{L}(G)$. We then have $s \in K^{\uparrow c}, \sigma \in \Sigma_{u c}$ and $s \sigma \in \mathcal{L}(G)$. Hence, because $K^{\uparrow c}$ is controllable w.r.t. $\Sigma_{u c}$ and $\mathcal{L}(G)$, $s \sigma \in K^{\uparrow c}$.

This proves that $K^{\uparrow c}$ is partially controllable w.r.t. $\Sigma_{i, u c}$, $\Sigma_{u c}, K_{i}, P_{i}^{-1}\left(\mathcal{L}\left(G_{i}\right)\right)$. Now, as $K_{i}^{\uparrow p c}$ is supremal and partially controllable w.r.t. $\Sigma_{i, u c}, \Sigma_{u c}, K_{i}, P_{i}^{-1}\left(\mathcal{L}\left(G_{i}\right)\right.$, we can deduce that $\forall i \leq n, K^{\uparrow c} \subseteq K_{i}^{\uparrow p c}$. Finally, $K^{\uparrow c} \subseteq \bigcap_{i \leq n} K_{i}^{\uparrow p c}$.

The interest of this method is that it avoids the building of the entire plant; hence reducing the complexity of the supervisory synthesis phase. Indeed, if $G=G_{1} \|$ $\cdots \| G_{n}$ is such that $\left|\mathcal{X}_{G_{i}}\right|=N$ and $H$, with $\left|\mathcal{X}_{H}\right|=$ $m$, is the FSM modeling the language specification $K$, then the FSM modeling the partial specification $K_{i}$ are in $\mathcal{O}(N . m)$. According to Section 3, the complexity to compute the supremal partially controllable sublanguage of each $K_{i}$ is in $\mathcal{O}\left(N^{2} . m\right)$. Finally, the overall complexity is in $\mathcal{O}\left(n . N^{2} . m\right)$. This has to be opposed to the space complexity $\mathcal{O}\left(N^{n} . m\right)$ of computing $(K \cap$ $\mathcal{L}(G))^{\uparrow c}$ on $G$, seen as a unique FSM.

Remark 1. To check that $K \subseteq \mathcal{L}(G)$ it is sufficient to check that $\forall 1 \leq i \leq n, K \subseteq P_{i}^{-1}\left(\mathcal{L}\left(G_{i}\right)\right)$. Hence, it is not necessary to compute $\mathcal{L}(G)$.

In some situations, modeling the expected behavior by a language included in the one of the plant may lead to a language that is too large to be efficiently represented. Moreover, requiring the inclusion of languages makes that the specification of $K$ may be itself relatively difficult to identify insofar as the language $\mathcal{L}(G)$ is not known. Theorem 4 gives another sufficient condition under which Theorem 2 gives access to the supremal 
solution. First, we need to introduce the notion of observable language.

Definition 4. Let $K$ and $M$ be two prefix-closed languages over $\Sigma$ and $\Sigma^{\prime}, \Sigma^{\prime \prime} \subseteq \Sigma$. $K$ is said to be observable w.r.t. $P_{\Sigma^{\prime}}, \Sigma^{\prime \prime}$ and $M$ if $\forall s, s^{\prime} \in K, \forall \sigma \in \Sigma^{\prime \prime}$, if $P_{\Sigma^{\prime}}\left(s^{\prime}\right)=P_{\Sigma^{\prime}}(s)$ and $s^{\prime} \sigma \in K$, and $s \sigma \in M$, then $s \sigma \in K$.

It is shown in e.g. [Cassandras and Lafortune (1999), Chap. 3.7] that the observability condition can be checked in $\mathcal{O}\left(m^{2} N\right)$, where $m$ and $N$ are the number of states of the FSM that generate $K$ and $M$.

Definition 5. Let $K \subseteq \Sigma^{*}$ be a prefix-closed language and $G=G_{1}\|\cdots\| G_{n}$ a concurrent system, then $K$ is said to be $G$-observable if $\forall i \in$ $\{1, \ldots, n\}, \forall s \in \Sigma^{*}, K_{i}\left(s, \Sigma_{u c}\right)$ is observable w.r.t. $P_{i}, \Sigma_{i, u c}$ and $P_{i}^{-1}\left(\mathcal{L}\left(G_{i}\right)\right)\left(s, \Sigma_{u c}\right)$, where $K_{i}=K \cap$ $\mathcal{L}\left(G_{i}\right), \forall i \leq n$.

The condition of $G$-observability states that after each trace $s$ admissible in $K_{i}$, if there exists two admissible suffixes of $s$ of uncontrollable events, say $s_{1}$ and $s_{2}$, that have the same projection over the local one (i.e. $\left.P_{i}\left(s_{1}\right)=P_{i}\left(s_{2}\right)\right)$, then if one can be extended by an uncontrollable event in a trace of $K_{i}$ and the other one in a trace of the abstracted specification $G_{i}^{-1}$, then the two extended traces have to belong to the specification. Finally, note that the $G$-observability condition can be checked in $\mathcal{O}\left(n m^{3} N^{4}\right)$, where $n$ is the number of components in $G, N$ the state size of $G_{i}$ and $m$ the size of the FSM that generates $K$. Now, based on Definition 5, we have that

Theorem 4. Assume that $\Sigma_{s} \subseteq \Sigma_{c}$ and that $K$ is $G$ observable, then $\bigcap_{i \leq n} K_{i}^{\uparrow p c}=(K \cap \mathcal{L}(G))^{\uparrow c}$

Proof : According to Theorem $2, \bigcap_{i \leq n} K_{i}^{\uparrow p c} \subseteq(K \cap$ $\mathcal{L}(G))^{\uparrow c}$ Thus, we have to show that

$$
\forall i \leq n,(K \cap \mathcal{L}(G))^{\uparrow c} \subseteq K_{i}^{\uparrow p c}
$$

To do so, let us consider $i \leq n$ and $\left((K \cap \mathcal{L}(G))^{\uparrow c}\right)^{\downarrow K_{i}, c}$ (the infimal prefix-closed and controllable superlanguage of $(K \cap \mathcal{L}(G))^{\uparrow c}$ w.r.t. $\Sigma_{u c}$ and $\left.K_{i}\right)$. We now prove that $\left((K \cap \mathcal{L}(G))^{\uparrow c}\right)^{\downarrow K_{i}, c}$ is partially controllable w.r.t $\Sigma_{i, u c}, \Sigma_{u c}, K_{i}$ and $P_{i}^{-1}\left(\mathcal{L}\left(G_{i}\right)\right)$. Indeed, if $\left((K \cap \mathcal{L}(G))^{\uparrow c}\right)^{\downarrow K_{i}, c}$ is partially controllable w.r.t $\Sigma_{i, u c}, \Sigma_{u c}, K_{i}$ and $P_{i}^{-1}\left(\mathcal{L}\left(G_{i}\right)\right)$, then we will have that $\left((K \cap \mathcal{L}(G))^{\uparrow c}\right)^{\downarrow K_{i}, c} \subseteq K_{i}^{\uparrow p c}$. Moreover, as $(K \cap \mathcal{L}(G))^{\uparrow c} \subseteq\left((K \cap \mathcal{L}(G))^{\uparrow c}\right)^{\downarrow K_{i}, c}$, we will also have that $(K \cap \mathcal{L}(G))^{\uparrow c} \subseteq K_{i}{ }^{\uparrow p c}$ and the proof will be done.

Let us now show that $\left((K \cap \mathcal{L}(G))^{\uparrow c}\right)^{\downarrow K_{i}, c}$ is partially controllable w.r.t $\Sigma_{i, u c}, \Sigma_{u c}, K_{i}$ and $P_{i}^{-1}\left(\mathcal{L}\left(G_{i}\right)\right)$. According to Definition 3, we have to show that $\left((K \cap \mathcal{L}(G))^{\uparrow c}\right)^{\downarrow} K_{i}, c$ is (i) controllable w.r.t. $\Sigma_{i, u c}$ and $P_{i}^{-1}\left(\mathcal{L}\left(G_{i}\right)\right)$ and (ii) controllable w.r.t. $\Sigma_{u c}$ and $K_{i}$. Item (ii) is obvious since by the definition of the infimal controllable language w.r.t. $\Sigma_{u c}$ and $K_{i}$, $\left((K \cap \mathcal{L}(G))^{\uparrow c}\right)^{\downarrow K_{i}, c}$ is controllable w.r.t. $\Sigma_{u c}$ and $K_{i}$. Let us now prove the point $(i)$
Let us consider $s \in\left((K \cap \mathcal{L}(G))^{\uparrow c}\right)^{\downarrow K_{i}, c}$ and $\sigma \in \Sigma_{i, u c}$ such that $s \sigma \in P_{i}^{-1}\left(\mathcal{L}\left(G_{i}\right)\right)$. We have

$$
s \sigma \in\left((K \cap \mathcal{L}(G))^{\uparrow c}\right)^{\downarrow K_{i}, c} . \Sigma_{i, u c} \cap P_{i}^{-1}\left(\mathcal{L}\left(G_{i}\right)\right)
$$

Moreover, $\left((K \cap \mathcal{L}(G))^{\uparrow c}\right)^{\downarrow K_{i}, c}=(K \cap \mathcal{L}(G))^{\uparrow c} . \Sigma_{u c}^{*} \cap$ $K_{i}$. So $s$ is of the form $s=s^{\prime} t$ with $s^{\prime} \in(K \cap \mathcal{L}(G))^{\uparrow c}$ and $t \in \Sigma_{u c}^{*}$. Now as $s \sigma=s^{\prime} t \sigma \in P_{i}^{-1}\left(\mathcal{L}\left(G_{i}\right)\right)$, we also have that $s^{\prime} P_{i}(t \sigma) \in P_{i}^{-1}\left(\mathcal{L}\left(G_{i}\right)\right)$ (Lemma 1).

We now have that $s^{\prime} \in(K \cap \mathcal{L}(G))^{\uparrow c}, P_{i}(t \sigma) \in$ $\Sigma_{i, u c}^{*}$ and $s^{\prime} P_{i}(t \sigma) \in P_{i}^{-1}\left(\mathcal{L}\left(G_{i}\right)\right)$. We then have that $s^{\prime} P_{i}(t \sigma) \in(K \cap \mathcal{L}(G))^{\uparrow c}$ as $(K \cap \mathcal{L}(G))^{\uparrow c}$ is controllable w.r.t. $\Sigma_{i, u c}$ and $P_{i}^{-1}\left(\mathcal{L}\left(G_{i}\right)\right)$ (Lemma 1). Finally, as $s^{\prime} P_{i}(t \sigma) \in(K \cap \mathcal{L}(G))^{\uparrow c} \subseteq K_{i}$, we obtain $P_{i}(t \sigma)=$ $P_{i}(t) \sigma \in K_{i}\left(s^{\prime}, \Sigma_{u c}\right)$.

Moreover, as by hypothesis $s \sigma=s^{\prime} t \sigma \in P_{i}^{-1}\left(\mathcal{L}\left(G_{i}\right)\right)$, we also have that $t \sigma \in P_{i}^{-1}\left(\mathcal{L}\left(G_{i}\right)\right)\left(s^{\prime}, \Sigma_{u c}\right)$. By definition of the projection, $P_{i}(t \sigma)=P_{i}\left(P_{i}(t \sigma)\right)$. Overall, we have that $P_{i}(t) \in K_{i}\left(s^{\prime}, \Sigma_{u c}\right), t \in K_{i}\left(s^{\prime}, \Sigma_{u c}\right)$, with $P_{i}(t)=P_{i}\left(P_{i}(t)\right), \sigma \in \Sigma_{u c}, P_{i}(t) \sigma \in K_{i}\left(s^{\prime}, \Sigma_{u c}\right)$ and $t \sigma \in P_{i}^{-1}\left(\mathcal{L}\left(G_{i}\right)\right)$. As $K_{i}\left(s^{\prime}, \Sigma_{u c}\right)$ is observable w.r.t. $P_{i}, \Sigma_{i, u c}$ and $P_{i}^{-1}\left(\mathcal{L}\left(G_{i}\right)\right)\left(s^{\prime}, \Sigma_{u c}\right)$, we obtain that $t \sigma \in K_{i}\left(s^{\prime}, \Sigma_{u c}\right)$. Hence $s \sigma\left(=s^{\prime} t \sigma\right) \in K_{i}$ and it entails that

$$
s \sigma \in\left((K \cap \mathcal{L}(G))^{\uparrow c}\right)^{\downarrow K_{i}, c} . \Sigma_{u c} \cap K_{i}
$$

As $\left((K \cap \mathcal{L}(G))^{\uparrow c}\right)^{\downarrow K_{i}, c}$ is (by definition) controllable w.r.t. $\Sigma_{u c}$ and $K_{i}, s \sigma \in\left((K \cap \mathcal{L}(G))^{\uparrow c}\right)^{\downarrow K_{i}, c}$. Thus $\left((K \cap \mathcal{L}(G))^{\uparrow c}\right)^{\downarrow K_{i}, c}$ is controllable w.r.t. $\Sigma_{i, u c}$ and $P_{i}^{-1}\left(\mathcal{L}\left(G_{i}\right)\right)$

Finally, $\left((K \cap \mathcal{L}(G))^{\uparrow c}\right)^{\downarrow K_{i}, c}$ is partially controllable w.r.t $\Sigma_{i, u c}, \Sigma_{u c}, K_{i}$ and $P_{i}^{-1}\left(\mathcal{L}\left(G_{i}\right)\right)$, which concludes the proof.

The complexity of checking the condition of $G$-observability and computing $(K \cap \mathcal{L}(G))^{\uparrow c}$ with our method is in $\mathcal{O}\left(n . m^{2} \cdot N^{2}\left(m . N^{2}+1\right)\right)$, which has to be opposed to $\mathcal{O}\left(N^{n} . m\right)$ (complexity with a monolithic approach).

The next proposition shows whenever $K$ is separable then it respects the conditions of Theorem 4.

Proposition 2. [Gaudin and Marchand (2004)] Let $G=$ $G_{1}\|\cdots\| G_{n}$ be the plant to be controlled s.t. $\mathcal{L}\left(G_{i}\right) \subseteq$ $\Sigma_{i}^{*}$. Let $K \subseteq \Sigma^{*}$ be the expected specification. If $K$ is separable w.r.t. $\left\{\Sigma_{i}\right\}_{1 \leq i \leq n}$ then $K$ is $G$-observable, i.e. $\forall i \leq n, \forall s \in K_{i}=K \cap P_{i}^{-1}\left(\mathcal{L}\left(G_{i}\right)\right), K_{i}\left(s, \Sigma_{u c}\right)$ is observable w.r.t. $P_{i}, \Sigma_{i, u c}$ et $P_{i}^{-1}\left(\mathcal{L}\left(G_{i}\right)\right)\left(s, \Sigma_{u c}\right)$. $\diamond$

The previous proposition states that whenever the specification is separable then our methodology offers an alternative way to the one of [Willner and Heymann (1991)] to compute $(K \cap \mathcal{L}(G))^{\uparrow c}$. Indeed, the solution of [Willner and Heymann (1991)] gives access to a set of decentralized supervisors acting upon each local component of the plant whereas, in our case, the result is a centralized supervisor (See Section 4.3 above).

A contrario, the next example shows that a language that respects the $G$-observability condition, may be not separable. 
Example 1. Let $G=G_{1} \| G_{2}$ with $\mathcal{L}\left(G_{1}\right)=$ $\overline{\left\{a_{1} \cdot u c_{1}\right\}}$ and $\mathcal{L}\left(G_{2}\right)=\overline{\left\{a_{2} \cdot u c_{2}\right\}}$. We have $\Sigma_{1, u c}=$ $\left\{u c_{1}\right\}$ and $\Sigma_{2, u c}=\left\{u c_{2}\right\}$. Let $K=\overline{\left\{a_{1} . u c_{1}, a_{2} . u c_{2}\right\}}$ be a prefix-closed language over $\Sigma=\Sigma_{1} \cup \Sigma_{2}$. One can check that $K$ verifies the $G$-observability condition. However, $K$ is not separable w.r.t. $\left\{\Sigma_{1}, \Sigma_{2}\right\}$, since $a_{1} \in$ $P_{1}(K)$ and $a_{2} \in P_{2}(K)$, thus $a_{1} a_{2} \in P_{1}(K) \| P_{2}(K)$ but $a_{1} a_{2} \notin K$.

\subsection{The Supervisor acting upon $G$.}

Let us now describe the way a supervisor can be extracted from the previously computed languages and how it can act upon $G$ in order to achieve the control objective $K$. With the notations of Theorem $2, \bigcap_{i \leq n} K_{i}^{\uparrow p c}$ is controllable with respect to $\Sigma_{u c}$ and $\mathcal{L}(G)$. However, it is not of interest to perform the intersection between these languages and to derive a supervisor from the result (all the computational advantages of our method would be lost). Following the concept of modularity described in [Wonham and Ramadge (1988)], the supervisor $\mathcal{S}$ will be seen as an oracle taking its decision according to the history of the system and the so-called pc-supervisors $\mathcal{S}_{i}^{p c}$ derived from $K_{i}^{\uparrow p c}$. The supervisor architecture is summarized in Figure 1. From each $K_{i}^{\uparrow p c}$, we derive a "supervisor" $\mathcal{S}_{i}^{p c}$, which after a trace of $G$ (which is also a trace of $P_{i}^{-1}\left(\mathcal{L}\left(G_{i}\right)\right)$ ), delivers the set of events that extend $s$ in a trace of $K_{i}^{\uparrow p c}$ (each of these pc-supervisor ensures on $P_{i}^{-1}\left(\mathcal{L}\left(G_{i}\right)\right)$ the partial controllability property w.r.t. $K$ and $\left.P_{i}^{-1}\left(\mathcal{L}\left(G_{i}\right)\right)\right)$. Further following the modularity concept, the set of allowed events is given by $\mathcal{S}(s)=\mathcal{S}_{1}^{p c}(s) \cap \cdots \cap \mathcal{S}_{n}^{p c}(s)$. Finally, the sub-set of events that is allowed in $G_{i}$ is given by $\mathcal{S}(s) \cap \Sigma_{i}$.

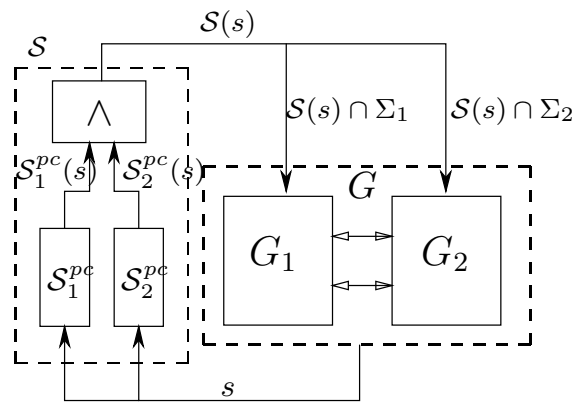

Fig. 1. Supervision Scheme

\section{CONCLUSION}

In this paper, we have investigated the Supervisory Control of Concurrent Discrete Event Systems. In particular, we proposed a modular method allowing to compute the supremal language of a specification $K$ controllable w.r.t. to the plant $G$. From the plant $G$ and each of its components $G_{i}$, we derive a set of approximations $\mathcal{L}\left(G_{i}^{-1}\right)$ and we ensure by control that each of these approximations respects a new language property, called partial controllability condition that depends on $K$. It is then shown that whenever the original specification respects some conditions (either $K \subseteq \mathcal{L}(G)$, or $K$ is $G$-observable) then a centralized supervisor can be extracted from the controlled approximations in such a way that the behavior of the controlled plant corresponds to the supremal controllable language contained in the one of the specification. Let us now emphasize some points that we did not mention so far but that are easy to deduce from the theorem $(2,3,4)$. If the specification is given in a modular way e.g. $K \cap K^{\prime}$, then in the case of prefix-closed languages, the modularity results in [Wonham and Ramadge (1988)] together with the results of Theorem 3, ensures that

$$
\bigcap_{i \leq n} K_{i}^{\uparrow p c} \cap \bigcap_{i \leq n} K_{i}^{\prime \uparrow p c}=\left[\left(K \cap K^{\prime}\right) \cap \mathcal{L}(G)\right]^{\uparrow c}
$$

as far as the conditions of Theorems 3 or 4 are satisfied by the two specifications.

If one want to change a component of $G$, e.g. replacing $G_{i}$ by $G_{i}^{\prime}$, then as far as $G_{i}^{\prime}$ is expressed using the same alphabet as the one of $G_{i}$ with the same partitioning between the controllable/uncontrollable event, then it is sufficient to recompute $K_{i}^{\prime \uparrow p c}=\left(K \cap P_{i}^{-1}\left(\mathcal{L}\left(G_{i}^{\prime}\right)\right)^{\uparrow p c}\right.$ in order to obtain the new supervisor (note that only the conditions referring to $G_{i}^{\prime}$ has to be (re)-checked). Hence this methodology is suitable for reconfigurable plants.

So far we have been interested in the control of plant for prefix-closed specification. We are currently looking for results ensuring that the controlled plant is non-blocking while still avoiding the computation of the whole state space. Another point of interest would be to extend these techniques to the hierarchical model described in [Gaudin and Marchand (2003)].

\section{REFERENCES}

S. Abdelwahed and W. Wonham. Supervisory control of interacting discrete event systems. In 41th IEEE Conference on Decision and Control, pages 1175-1180, Las Vegas, USA, December 2002.

K. Akesson, H. Flordal, and M. Fabian. Exploiting modularity for synthesis and verification of supervisors. In Proc. of the IFAC, 2002.

C. Cassandras and S. Lafortune. Introduction to Discrete Event Systems. Kluwer Academic Publishers, 1999.

M.H. deQueiroz and J.E.R. Cury. Modular supervisory control of large scale discrete-event systems. In Discrete Event Systems: Analysis and Control. Proc. WODES'00, pages 103-110, 2000.

B. Gaudin and H. Marchand. Modular supervisory control of asynchronous and hierarchical finite state machines. In European Control Conference, ECC 2003, Cambridge, UK, September 2003.

B. Gaudin and H Marchand. Supervisory control of concurrent discrete event systems. Research Report 1593, IRISA, February 2004. available at http://www.irisa.fr/vertecs/Publis/Ps/1593.ps.

S. Jiang and R. Kumar. Decentralized control of discrete event systems with specializations to local control and concurrent systems. IEEE Transactions on Systems, Man, and Cybernetics, Part B, 30(5):653660, October 2000.

R.J. Leduc, B.A. Brandin, W.M. Wonham, and M. Lawford. Hierarchical interface-based supervisory control: Serial case. In Proc. of 40th Conf. Decision Contr., pages 4116-4121, December 2001.

P. J. Ramadge and W. M. Wonham. The control of discrete event systems. Proceedings of the IEEE; Special issue on Dynamics of Discrete Event Systems, 77(1):81-98, 1989.

K. Rohloff and S. Lafortune. The control and verification of similar agents operating in a broadcast network environment. In 42nd IEEE Conference on Decision and Control, Hawaii, USA, 2003.

Y. Willner and M. Heymann. Supervisory control of concurrent discrete-event systems. International Journal of Control, 54(5): 1143-1169, 1991.

W. M. Wonham and P. J. Ramadge. Modular supervisory control of discrete event systems. Mathematics of Control Signals and Systems, 1:13-30, 1988. 